\title{
Influence of Collision Cascade Statistics on Pattern Formation of Ion-Sputtered Surfaces
}

\author{
M. Feix, A. K. Hartmann $*$ and R. Kree母 \\ Institut für Theoretische Physik, Universität Göttingen, Göttingen, Germany

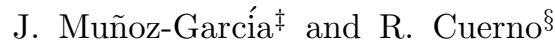 \\ Departamento de Matemáticas and Grupo Interdisciplinar de Sistemas Complejos (GISC), \\ Universidad Carlos III de Madrid, Avenida de la Universidad, 30, 28911 Leganés, Spain
}

(Dated: February 2, 2008)

\begin{abstract}
Theoretical continuum models that describe the formation of patterns on surfaces of targets undergoing ion-beam sputtering, are based on Sigmund's formula, which describes the spatial distribution of the energy deposited by the ion. For small angles of incidence and amorphous or polycrystalline materials, this description seems to be suitable, and leads to the classic BH morphological theory [R. M. Bradley and J. M. E. Harper, J. Vac. Sci. Technol. A 6, 2390 (1988)]. Here we study the sputtering of $\mathrm{Cu}$ crystals by means of numerical simulations under the binary-collision approximation. We observe significant deviations from Sigmund's energy distribution. In particular, the distribution that best fits our simulations has a minimum near the position where the ion penetrates the surface, and the decay of energy deposition with distance to ion trajectory is exponential rather than Gaussian. We provide a modified continuum theory which takes these effects into account and explores the implications of the modified energy distribution for the surface morphology. In marked contrast with BH's theory, the dependence of the sputtering yield with the angle of incidence is non-monotonous, with a maximum for non-grazing incidence angles.

PACS numbers: 05.10.-a, 68.35.-p, 79.20.-m
\end{abstract}

\section{INTRODUCTION}

Ion bombardment of solids often gives rise to characteristic surface topographies, which evolve under stationary and homogeneous ion fluxes. Besides kinetic roughening, wavelike ripple structures may occur. Such height modulations on the submicron scale have been observed for crystalline semiconductors 1.2 as well as for crystalline metals ${ }^{3.4}$ and some amorphous $s^{\frac{5}{3}}$ and polycrystalline materials, see a recent review in Ref. 6. According to continuum theories, which are based on the work of Bradley and Harper $(\mathrm{BH}) \stackrel{7}{\underline{T}}$ the periodic patterns emerge from a competition between a roughening curvature instability due to characteristics of the spreading of ion energy, and simultaneous smoothing processes due to surface diffusion ${ }^{8.9}$ Although this mechanism seems to be quite universal, there are materialspecific differences in the evolution of surface topographies. For non-metallic substrates, for example, one usually needs off-normal incidence of ion flux to produce ripples, which change their orientation with the incidence angle 2.4.5.10.11.12.13.14 whereas ripples are observed on metallic substrates even at normal incidence, and the orientation of ripples changes with substrate temperature ${ }^{3.15 .16}$ Furthermore, the smoothing mechanism of surface diffusion is not well understood yet. In previous simulations, $\frac{17}{17}$ we have found that the emerging patterns depend crucially on the diffusion mechanisms applied. In particular the long-time behavior, which is governed in the continuum theory by non-linear terms, depends even qualitatively on the surface diffusion mechanism. Given that the surface topographies resulting from different mechanisms of surface diffusion have been studied by simulations elsewhere,, 18 in the present work we will focus on specificities due to the energy deposition process.

Continuum theories for the surface morphology of the target usually assume that the kinetic energy of an ion hitting a solid surface spreads in the bulk and produces a Gaussian density of deposited energy

$$
\begin{aligned}
\epsilon_{s}(\mathbf{r}) & =N_{s} \epsilon e^{-\frac{x^{2}+y^{2}}{2 \beta^{2}}} e^{-\frac{(z+a)^{2}}{2 \alpha^{2}}}, \\
N_{s} & =\left[(2 \pi)^{3 / 2} \alpha \beta^{2}\right]^{-1},
\end{aligned}
$$

where $\mathbf{r}=(x, y, z)$ is a point within the target, ions are falling along the $\hat{\mathbf{z}}$ axis and penetrate an average distance $a$ within the solid, $\epsilon$ is the average kinetic energy carried by each ion, and the values of $\alpha, \beta$ describe the spreading of the energy, they are of the same order of magnitude as $a$. The Gaussian form (1) is based on the work of Sigmund, 19 who considered a polycrystalline or amorphous target and analyzed the kinetic transport theory of the sputtering process. He found that in the elastic collision regime at energies where electronic stopping is not dominating, the deposited energy can be approximated by a Gaussian near its maximum. The quality of the approximation is reasonable, if mass differences between substrate and ion are not too large. Obviously, the Gaussian form is not universal and consequences of deviations from the Gaussian form within the BH model have not been studied yet. In particular, although the observations of ripples on single crystalline metals ${ }^{3.15 .16}$ are qualitatively described by the $\mathrm{BH}$ model, the latter is strictly a theory for amorphous materials, and thus there 
is a need to justify theoretically the emergence of such type of patterns onto this other class of substrates.

Obtaining more detailed information about the deposited energy from simulations has become straightforward by now, as there are many well calibrated, efficient simulation methods for ion impact available ${ }^{20,21,22,23.24}$ In the present work, we use simulations based on the binary collision approximation ${ }^{22.24 .25}$ and consider a metallic material $(\mathrm{Cu})$, for which we generate statistical ensembles of collision cascades emerging from single ion impact events on plane surfaces. We analyze the data in terms of deposited energies as well as ejected particles. We do not claim to perform the best state of the art simulation of ion impact on $\mathrm{Cu}$ (for example, we only consider a very rough model of surface binding forces). Rather, we aim at more generic results, which are of relevance to the theory of surface evolution. Our simulations provide an average density of deposited energy, which is quite different from the Gaussian form in Eq. (11). We furthermore consider the fluctuations around this average and find strong, intrinsic noise. In the subsequent part of our work we investigate the consequences of the simulation results for the continuum theory of pattern formation by ion-beam sputtering. We obtain that the modified energy distribution obtained in the numerical simulations induces a sputtering yield that overcomes some of the shortcomings (when comparing with experiments) of the analogous result within BH's theory. Moreover, we recover the production of the ripple instability, and the dependence of the pattern features with phenomenological parameters similar to $\mathrm{BH}$ theory, thus providing a theoretical framework within which observations of ripples on metals can be naturally accomodated $\stackrel{3.15 .16}{ }$

\section{OBSERVABLES OF CASCADE STATISTICS}

In this section we want to relate observables of our simulations to the phase space density

$$
g\left(\boldsymbol{v}, \boldsymbol{\rho}, z, t \mid \boldsymbol{\rho}_{0}, z=0, \boldsymbol{v}_{0}, t=0\right),
$$

where $\boldsymbol{\rho} \equiv(x, y)$. This function is the basic quantity underlying the kinetic theory of collision cascades and also introduces the quantities which are used in the construction of a continuum theory of surface pattern formation by ion bombardment. Function $g$ is the average density of cascade particles in 6-dimensional $(\boldsymbol{v}, \boldsymbol{\rho}, z)$ space at time $t$, under the assumption that one ion has hit the surface at $\boldsymbol{\rho}_{0}$ and at $t=0$ with velocity $\boldsymbol{v}_{0}$. As we will only treat identical initial conditions with $\boldsymbol{\rho}_{0}=0$ and $\boldsymbol{v}_{0}=-\left|\sqrt{2 \epsilon_{0} / m}\right| \boldsymbol{e}_{z}$, we will use the abbreviated notation $g(\boldsymbol{\rho}, z, \boldsymbol{v}, t)$ and drop the explicit dependence on $\boldsymbol{\rho}_{0}$ and $\boldsymbol{v}_{0}$. The average has to be taken over an ensemble of targets, which differ by random, thermal displacements of atoms.

To define our simulation observables in terms of the phase space density, first note that $g(\boldsymbol{\rho}, z, \boldsymbol{v}, t)(\boldsymbol{v}$. da) $d^{3} \boldsymbol{v} d t$ is the number of particles, which penetrate a surface element $d \boldsymbol{a}$ situated at position $(\boldsymbol{\rho}, z)$, with velocity $\boldsymbol{v}$ during the time interval $d t$.

The phase space density and the corresponding current density may as well be considered as functions of position, energy and direction of velocity using $\boldsymbol{v}=\sqrt{2 \epsilon / m} \hat{\boldsymbol{v}}, \hat{\boldsymbol{v}}$ being the unit vector in the direction of $\boldsymbol{v}$, so that $\boldsymbol{v} d^{3} \boldsymbol{v}=$ $\left(2 / m^{2}\right) \epsilon \hat{\boldsymbol{v}} d \epsilon d \hat{\boldsymbol{v}}$. The current density $\boldsymbol{j}(\epsilon, \boldsymbol{\rho}, z, t) d \epsilon$ of cascade particles of energy near $\epsilon$ is given by

$$
\boldsymbol{j}(\epsilon, \boldsymbol{\rho}, z, t) d \epsilon=\frac{2}{m^{2}} d \epsilon \int d \hat{\boldsymbol{v}} \epsilon \hat{\boldsymbol{v}} g(\epsilon, \hat{\boldsymbol{v}}, \boldsymbol{\rho}, z, t) .
$$

If we restrict the integration over $\hat{\boldsymbol{v}}$ to directions with $\hat{\boldsymbol{v}} \cdot d \boldsymbol{a}>0$, we only count particles, which cross the surface element in the direction of its normal. This variant will be denoted by $\boldsymbol{j}^{+}$.

From Eq. (3) it is obvious that the time integral

$$
h_{2 d}(\epsilon, \boldsymbol{\rho}, z) d x d y=\int_{0}^{\infty} d t \boldsymbol{e}_{z} \cdot \boldsymbol{j}(\epsilon, \boldsymbol{\rho}, z, t) d x d y
$$

equals the total average number of particles per energy at energy $\epsilon$ of a single collision cascade, which penetrate the surface element $d x d y \boldsymbol{e}_{z}$ located at $(\boldsymbol{\rho}, z)$.

Note that $h_{2 d}$ is a surface density and the quantities

$$
n_{2 d}(\boldsymbol{\rho}, z)=\int_{0}^{\infty} d \epsilon h_{2 d}(\epsilon, \boldsymbol{\rho}, z)
$$

and

$$
e_{2 d}(\boldsymbol{\rho}, z)=\int_{0}^{\infty} d \epsilon \epsilon h_{2 d}(\epsilon, \boldsymbol{\rho}, z)
$$

give the average number of particles per area and average energy per area transported to the $(x y)$-plane at $z$ by the collision cascade. For all these quantities the corresponding variants $h_{2 d}^{+}, n_{2 d}^{+}, e_{2 d}^{+}$only take into account particles moving in outward direction $+\boldsymbol{e}_{z}$.

The particles arriving at the $z=0$ plane (which constitutes the surface of the material) with velocities in outward direction will leave the bulk if they overcome the surface binding forces. We will use a simple spherical barrier model of surface binding with barrier height $U$. This implies that all particles arriving at the surface with kinetic energy $\epsilon>U$ will be sputtered off. The surface density $n_{U}$ of these particles is therefore given by Eq. (5) with the lower boundary of the $\epsilon$ integration replaced by $U$. The total sputtering yield is the surface integral of this density, $Y_{U}=\int d^{2} \boldsymbol{\rho} n_{U}(\boldsymbol{\rho})$. At internal surfaces there is no surface binding and thus

$$
p(\epsilon, \boldsymbol{\rho}, z)=h_{2 d}(\epsilon, \boldsymbol{\rho}, z) / Y_{U=0}
$$

becomes the probability density to find a particle with energy $\epsilon$ crossing the internal surface at location $(\boldsymbol{\rho}, z)$ and $p_{U}(\boldsymbol{\rho})=n_{U}(\boldsymbol{\rho}) / Y_{U}$ is the probability density to find a particle leaving the bulk at $\boldsymbol{\rho}$. These are the quantities we will study in the subsequently described simulations. 


\section{BCA SIMULATIONS}

Atomic displacements and particle ejection from a solid due to the impact of a single ion with kinetic energy in the $\mathrm{keV}$ range can be simulated by using the binary collision approximation 24 (BCA). The basic idea is to substitute the detailed particle trajectories by trajectories where the particles travel with constant velocity until they "hit" onto another particle. Each collision event is integrated analytically or numerically, leading to new positions and velocities of the particles participating in the collision. Hence the full dynamical process is reduced to a cascade of collisions. A sample cascade, originating from an impact of a $5 \mathrm{keV} \mathrm{Cu}$ ion on a $\mathrm{Cu}$ lattice with an angle of incidence of $60^{\circ}$, is shown in Fig. 11 Although this method has its limitations, 24 it has become a standard technique and is used to describe ion implantation and sputtering.

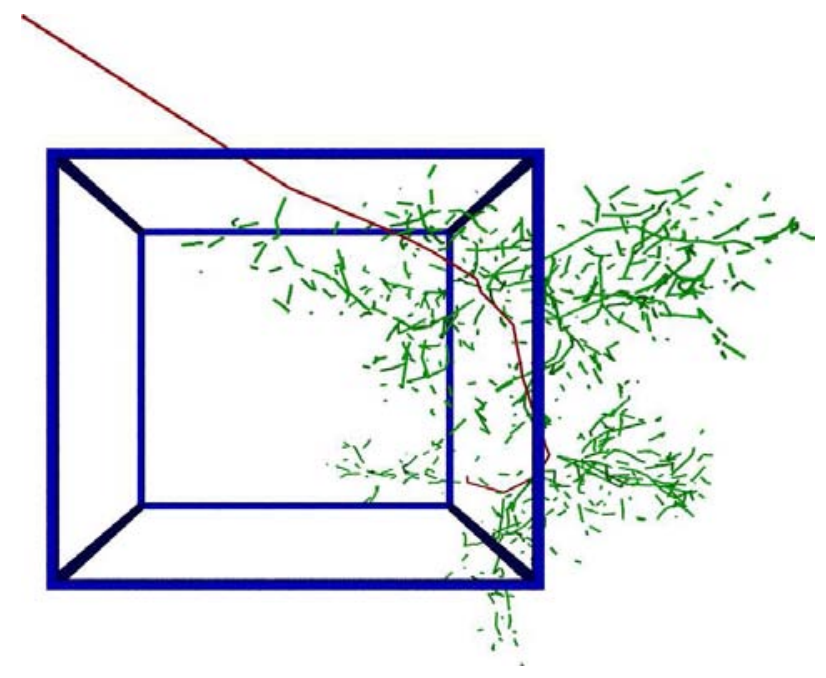

FIG. 1: Sample cascade originating from an impact of a 5 $\mathrm{keV} \mathrm{Cu}$ ion on a $\mathrm{Cu}$ crystal. The angle of incidence is $60^{\circ}$. The cube shown acts just as scale and has size $2.65 \mathrm{~nm}^{3}$, while the full lattice simulated has size $(10.6 \mathrm{~nm})^{2} \times 18 \mathrm{~nm}$.

We have performed BCA simulations of single ion impact on a plane $\mathrm{Cu}$-surface at normal incidence with velocity $\boldsymbol{v}_{0}=-\left|\boldsymbol{v}_{0}\right| \boldsymbol{e}_{z}$.

All the statistical information was obtained from ensembles of 3000-6000 ion impacts per ensemble, which we generated for a single initial condition of the ion. The positions of the atoms making up the undisturbed solid were displaced from ideal lattice sites of a $\mathrm{Cu}$ single crystal (170000 atoms of an fcc structure with lattice constant $3.61 \AA$ corresponding to a solid of $10.6 \mathrm{~nm} \times 10.6$ $\mathrm{nm}$ and a bulk depth of $18 \mathrm{~nm}$ ) by uncorrelated, Gaussian distributed displacements to account for thermal fluctuations. For each ion of an ensemble, an additional homogeneous lateral random displacement was added, which was taken to be uniformly distributed within a square of edge length 1 lattice constant. Thus within every ensemble the ion hits upon macroscopically identical but microscopically differing configurations of the solid.

We considered two orientations of the crystal, $(1,0,0)$ and $(58,72, \overline{39})$. The latter was used to suppress effects of crystal anisotropy and we found very good agreement between the angular averages of $n_{2 d}, e_{2 d}$ obtained from $(1,0,0)$ and the corresponding quantities obtained from the oblique orientation.

Our BCA code follows standard implementations. ${ }^{22}$ It allows for arbitrary positions of the bulk atoms and is suitable for studying defect accumulation during multiple impact (although this feature is not used in the present work). Simple and well-tested forms of the screened Coulomb potential ${ }^{26}$ and the inelastic processes 22.27 have been chosen. All model parameters of the algorithm have been adjusted to $\mathrm{Cu}$ projectiles of a few $\mathrm{keV}$, hitting a $\mathrm{Cu}$ single crystal. 28 These choices allowed for easy calibration and comparisons of our implementation against literature results. It should be emphasized, however, that the main focus of the present work is on generic results, which are of relevance for pattern formation of ion-sputtered surfaces of metals.

\section{SIMULATION RESULTS}

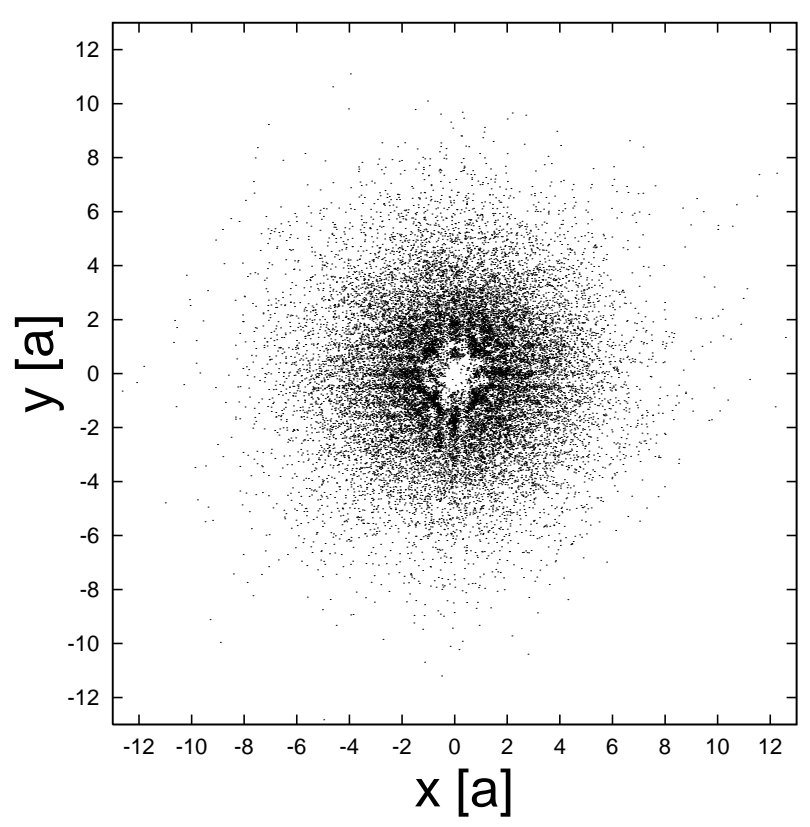

FIG. 2: Spatial distribution of ejected $\mathrm{Cu}$ atoms emerging from 6000 independent trials of hitting the $(x, y)$ crystal surface (oriented in $(1,0,0)$ direction) with a single $5 \mathrm{keV} \mathrm{Cu}$ ion at normal incidence. Distances are measured in units of $a=3.61 \AA$.

Fig. 2 shows the surface distribution of all the ejected particles within an ensemble of 6000 cascades, each emerging from one incident $5 \mathrm{keV} \mathrm{Cu}$ ion (normal incidence) for the crystal in $(1,0,0)$ orientation. Clearly, a 
"hole" around the location of impact is visible. This is in contrast to what can be expected when applying Eq. (1). The scattering is almost rotational invariant, a slight $90^{\circ}$ rational-invariant structure is visible, reflecting the lattice structure. To check whether the result is an artefact of the crystal orientation, we studied also a $(58,32, \overline{39})$ surface, see Fig. 3. Although the plot exhibits slightly less structures, again only few particles are ejected near the point of penetration. Hence, we decided to concentrate on the oblique $(58,32, \overline{39})$ orientation, because we want to study generic results irrespective of specific crystal orientations. In any case, for both surface orientations and small values of $\Delta$, the angular average of data obtained from

$$
n_{2 d}(\rho)=\frac{1}{2 \pi \rho \Delta} \int_{0}^{2 \pi} d \phi \int_{\rho}^{\rho+\Delta} \rho d \rho n_{2 d}(\rho, \phi),
$$

where $\rho=|\boldsymbol{\rho}|$, turns out to be nearly undistinguishable.

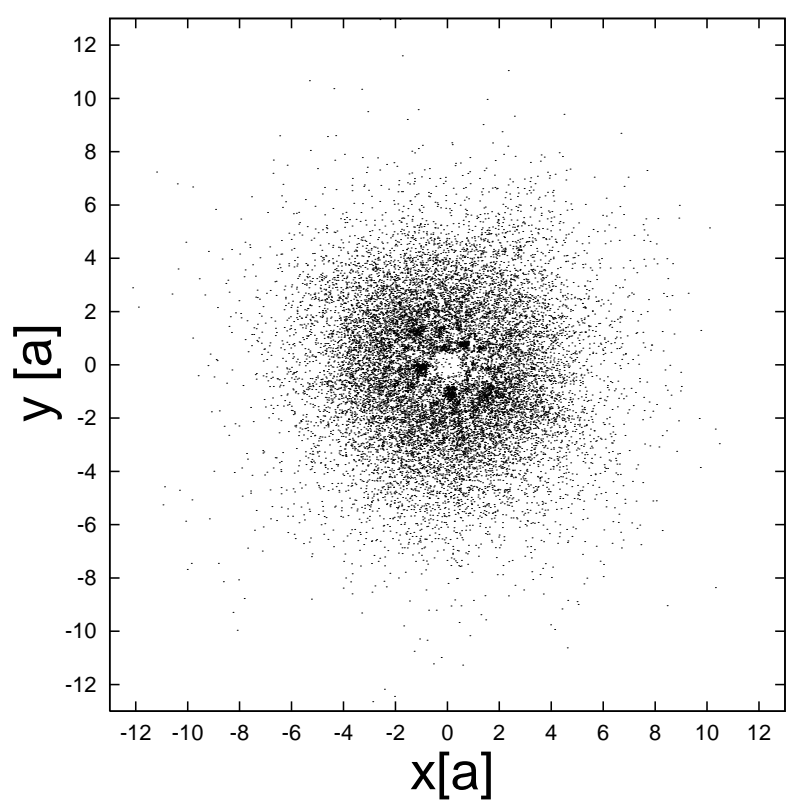

FIG. 3: Spatial distribution of ejected $\mathrm{Cu}$ atoms emerging from 6000 independent trials of hitting the $(x, y)$ crystal surface (oriented in $(58,32, \overline{39})$ direction) with a single $5 \mathrm{keV} \mathrm{Cu}$ ion at normal incidence. Distances are measured in units of $a=3.61 \AA$.

Fig. 4 shows the corresponding probability density $p(\rho)$ per surface unit, averaged over all angles, of finding an ejected particle a distance $\rho$ from the point of incidence of the $\mathrm{Cu}$ ion. This figure shows that the assumption on the ejection probability being distributed following a Gaussian distribution, hence leading to a maximum at $\rho=0$, is not justified in case of crystals. This is in contrast to amorphous materials, where a more Gaussian-like distribution of the ejection probability has been observed 29 when using the simulation packet SRIM with the same ion/bulk parameters as above.

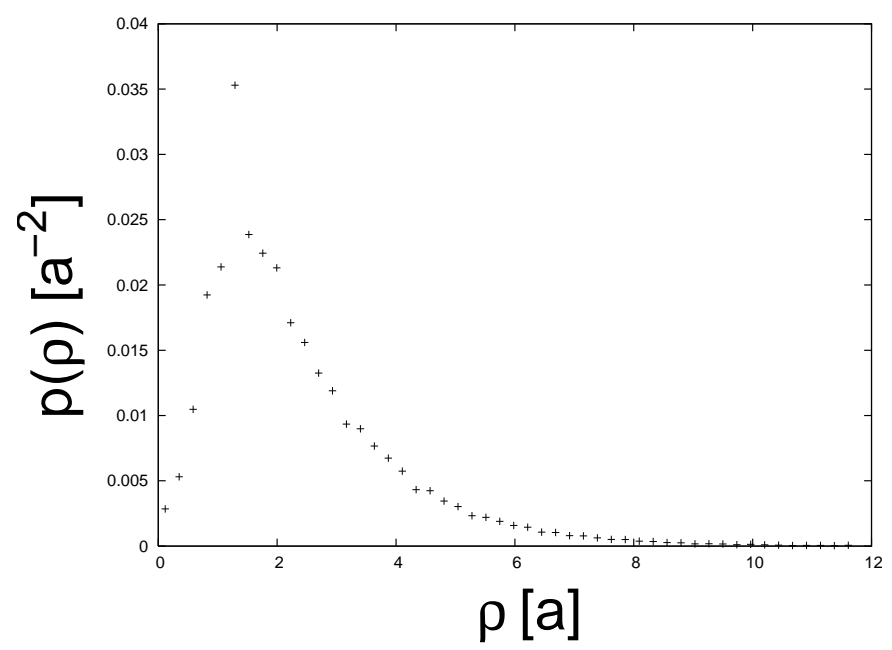

FIG. 4: Probability of ejected particles vs distance $\rho$ from point of ion incidence (measured in units of $a=3.61 \AA$ ) determined from the data of Fig. (3).

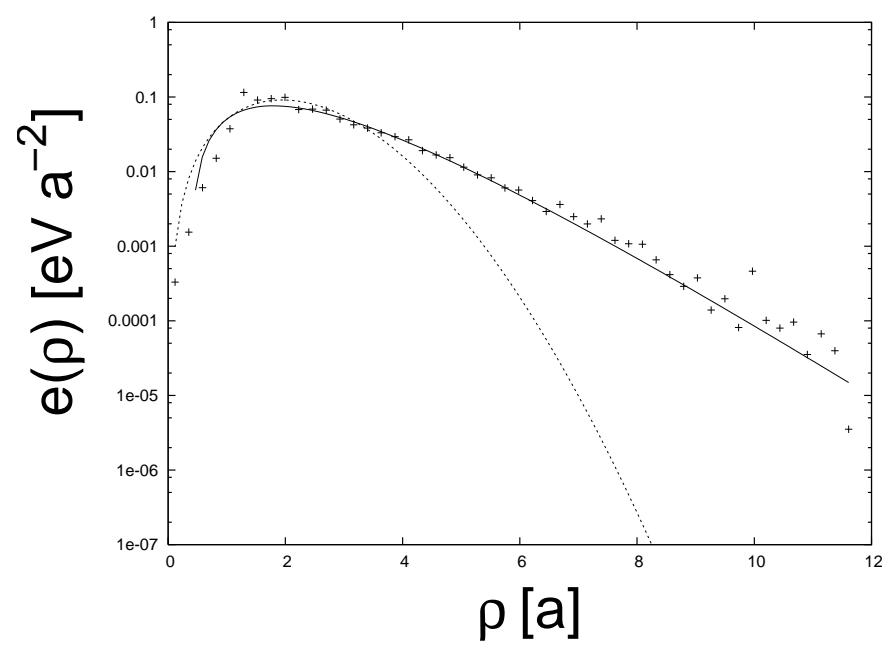

FIG. 5: Surface density of mean energy of sputtered $\mathrm{Cu}$ atoms vs distance $\rho$ (measured in units of $a=3.61 \AA$ ) from point of ion incidence for $5 \mathrm{keV} \mathrm{Cu}$ ions on semi-log scale. The solid line is the best fit of the data to an exponential with a polynomial prefactor and corresponds to $0.297\left(\rho^{2}-\right.$ $0.392 \rho) \exp (-1.27 \rho)$. The dotted line, which corresponds to a fit to a Gaussian, is obviously inadequate.

Fig. 5 displays the corresponding angular average of the surface density $e_{2 d}(\rho)$ of the energy of sputtered particles. In this figure, we have also shown two MarquardtLevenberg fits $\left(f_{s}=\left(a \rho^{2}+b \rho\right) \exp \left[-c \rho^{s}\right]\right.$ with $s=1$ and $s=2$ ) to the data. One can see that the decay of the energy density is not in accordance with a Gaussian, even when including a decay towards the point $\rho=0$ of penetration, as suggested by Eq. (1). The data can be fitted well to a exponential decay with a simple polynomial prefactor.

In the previous figure, we have studied the mean, hence 
let us now consider characteristic features of the probability density $p(\rho, \epsilon)$. In Fig. [6] we show the surfaceintegrated probability density $p(\epsilon)=\int d^{2} \boldsymbol{\rho} p(\epsilon, \rho)$. The behavior is remarkable close to a simple power law

$$
p(\epsilon) \approx \frac{a}{(b+\epsilon)^{\alpha}} \sim \epsilon^{-2}
$$

outside of a region of small $\epsilon$. Our best fit corresponds to $a=5.26, b=5.03, \alpha=1.87$.

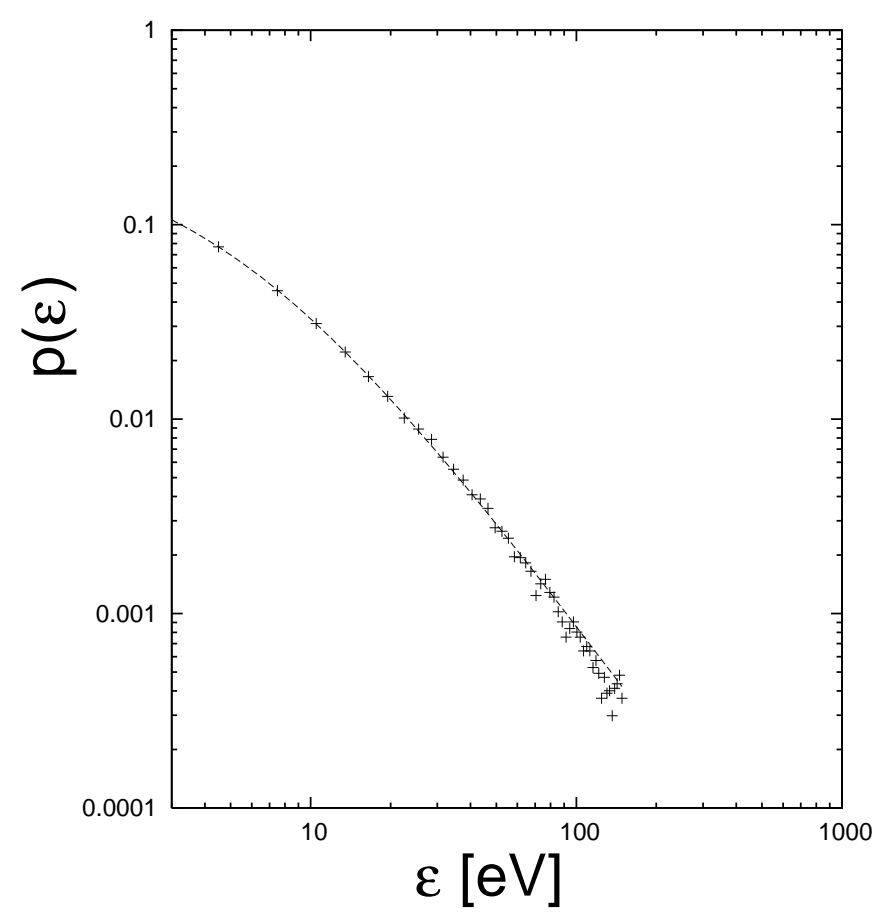

FIG. 6: Probability density of energy, which is transported to the surface by a single collision cascade emerging from a 5 $\mathrm{keV} \mathrm{Cu}$ ion on a log-log scale. The solid line corresponds to $5.259(5.035+\epsilon)^{-1.874}$, which is the best fit to a simple power law $a /(b+\epsilon)^{\alpha}$.

Fig. 7 displays the conditional probability density $p(\epsilon \mid \rho)$ of energy at fixed $\rho$ for different values of $\rho$. Surprisingly, the conditional density does not depend on $\rho$ significantly. This shows that

$$
p(\epsilon, \rho) \approx p(\rho) p(\epsilon) \propto \frac{a n_{2 d}(\rho)}{(b+\epsilon)^{2}}
$$

outside of a region of very small distances. An immediate implication is that

$$
e_{2 d}(\rho) \propto n_{2 d}(\rho)
$$

so that the number of ejected particles and the energy deposited at the surface are proportional to each other, as assumed in the BH theory. However, another important implication is that the amount of energy transported to the surface is subjected to strong internal noise, which may limit the applicability of deterministic continuum

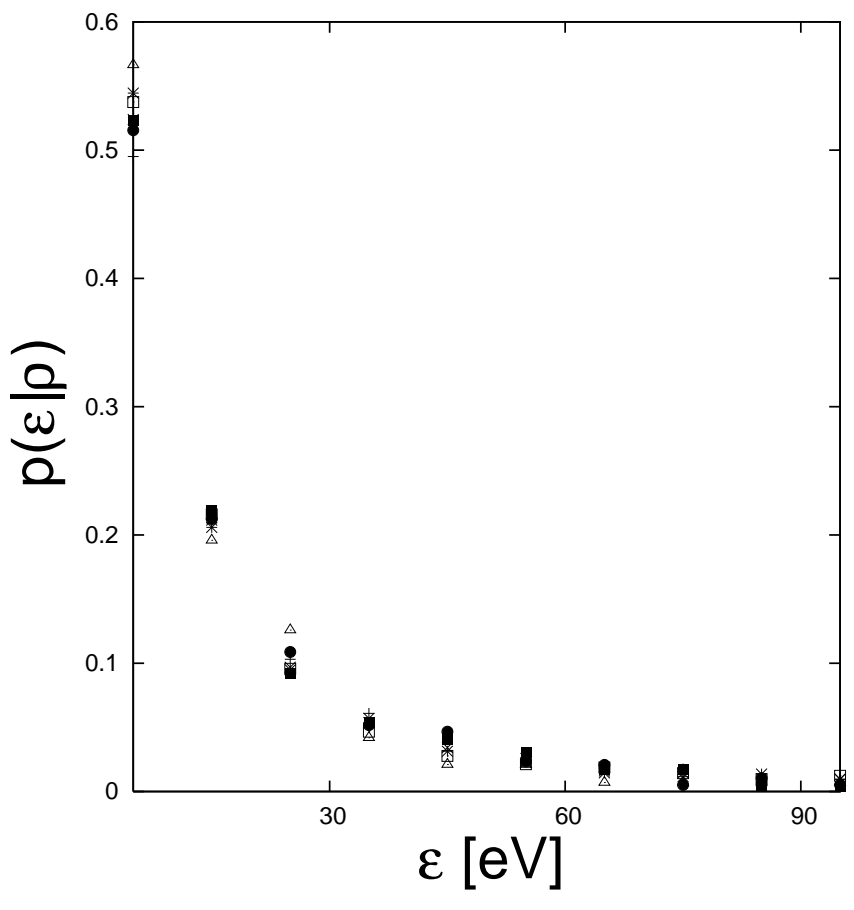

FIG. 7: Conditional probability density $p(\epsilon \mid \rho)$ to find energy $\epsilon$ of ejected particles keeping the distance $\rho$ fixed. Different symbols correspond to different values $\rho=1.759,2.914,4.104,5.277,6.450,7.623,8.795,9.968,11.141$ As the data for different $\rho$ are almost undistinguishable, differences not being significant, we need not provide a detailed legend.

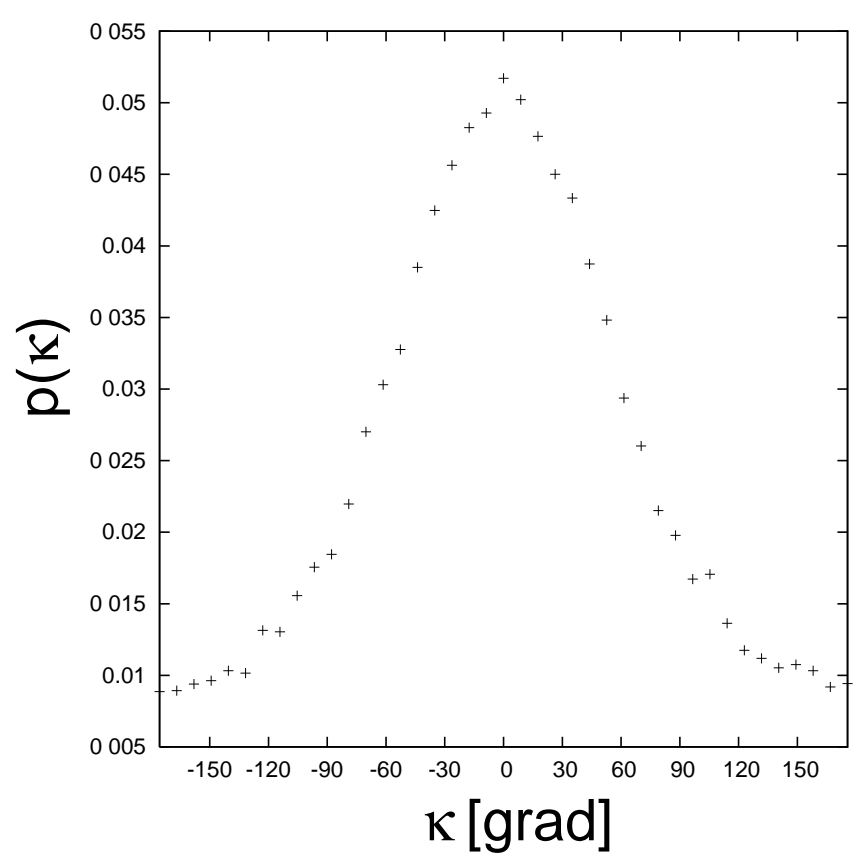

FIG. 8: Distribution of ejected particles with different directions of velocity. Here $\kappa$ denotes the angle between the projection of particle velocity onto the surface and the vector between point of ion impact and point of particle ejection. 
theories based on the average energy at the surface. This problem will be pursued elsewhere.

To gain further insight into the structure of the ensemble of cascade particles at the surface, we have also studied correlations between the position $\boldsymbol{\rho}$ and the projection of velocity, of sputtered particles onto the surface, $\boldsymbol{v}_{\text {surf }}=\boldsymbol{v}-\left(\boldsymbol{v} \cdot \boldsymbol{e}_{z}\right) \boldsymbol{e}_{z}$. Fig. 8 shows the distribution of the angle $\kappa$ between $\boldsymbol{\rho}-\boldsymbol{\rho}_{0}$ and $\boldsymbol{v}_{\text {surf }}$, where $\boldsymbol{\rho}_{0}$ is the point at which the ion hits the surface and $\rho$ is the position of a cascade particle arriving at the surface with velocity $\boldsymbol{v}$. The figures shows that, for typical collision cascades, most of the ejected particles move away from the point of ion impact.

\section{CONTINUUM APPROXIMATION TO ENERGY DEPOSITION}

Within Sigmund's approximation, 30 the rate at which the target is being eroded at an arbitrary point on the surface, is proportional to the total amount of energy deposited there from ion collisions. In his theory for amorphous or polycrystalline targets, an accurate description of the sputtering phenomena can be achieved by assuming that energy is deposited following the Gaussian distribution (11).

Bradley and Harper $(\mathrm{BH})^{7}$ later employed this energy distribution in order to compute the local erosion velocity at an arbitrary surface point $O$, allowing for gentle surface undulations. To perform the calculation, a new local reference frame is taken in which the $\hat{\mathbf{z}}^{\prime}$ axis is taken along the surface normal at $O$. The principal curvatures are assumed along the $\hat{\mathbf{x}}^{\prime}$ and $\hat{\mathbf{y}}^{\prime}$ axes, that are defined, respectively, as the direction orthogonal to $\hat{\mathbf{z}}^{\prime}$ that is in the plane defined by this axis and the ion trajectory and the remaining direction in order to make up a right-handed reference frame. Assuming that the radii of curvature at $O, R_{x}$ and $R_{y}$, are much larger than the penetration depth $a$, the surface height can be approximated to $z^{\prime}\left(x^{\prime}, y^{\prime}\right)=-\frac{1}{2}\left(\frac{x^{\prime 2}}{R_{x}}+\frac{y^{\prime 2}}{R_{y}}\right)$. In order to obtain the erosion velocity, we have to add up the total energy deposited at $O$ from ions entering the whole target, expressing the ion flux and energy distribution in the latter reference frame, which is related with the one implicit in (10) as

$$
\begin{aligned}
& \hat{\mathbf{x}}=\hat{\mathbf{x}}^{\prime} \cos \left(\gamma_{0}\right)+\hat{\mathbf{z}}^{\prime} \sin \left(\gamma_{0}\right), \\
& \hat{\mathbf{y}}=\hat{\mathbf{y}}^{\prime} \\
& \hat{\mathbf{z}}=\hat{\mathbf{z}}^{\prime} \cos \left(\gamma_{0}\right)-\hat{\mathbf{x}}^{\prime} \sin \left(\gamma_{0}\right),
\end{aligned}
$$

with $\gamma_{0}$ being the incidence angle formed between the ion trajectories and the surface normal at $O$. Accounting up to curvature corrections, the ion flux reads $\Phi\left(x^{\prime}, y^{\prime}\right)=$ $\Phi_{0} \cdot\left(\cos \gamma_{0}-\frac{x^{\prime}}{R_{x}} \sin \gamma_{0}\right)$, where $\Phi_{0}$ is the constant nominal ion flux. Taking all this into account, the erosion velocity at $O$ reads, finally,

$v_{O}\left(\gamma_{0}, R_{x}, R_{y}\right)=\Lambda \int_{-\infty}^{+\infty} \int_{-\infty}^{+\infty} \Phi\left(x^{\prime}, y^{\prime}\right) \epsilon_{s}\left(x^{\prime}, y^{\prime}\right) d x^{\prime} d y^{\prime}$, where $\Lambda$ is a proportionality constant relating deposited energy with the number of sputtered atoms, and the integration limits are taken to infinity thanks to the fast decay of the energy distribution $\epsilon_{s}=e_{2 d}$. By expanding (13) to lowest non-trivial order in $a / R_{x}, a / R_{y} \ll 1$, Bradley and Harper obtained ${ }^{7}$

$$
v_{O}=N_{s} \Lambda \epsilon \Phi_{0} e^{-\frac{a^{2}}{2 \alpha^{2}}}\left[\Gamma_{0}\left(\gamma_{0}\right)+\frac{\Gamma_{x}\left(\gamma_{0}\right)}{R_{x}}+\frac{\Gamma_{y}\left(\gamma_{0}\right)}{R_{y}}\right],
$$

where $\Gamma_{0}\left(\gamma_{0}\right), \Gamma_{x}\left(\gamma_{0}\right)$, and $\Gamma_{y}\left(\gamma_{0}\right)$ are functions that depend on the incidence angle $\gamma_{0}$, but also on features of the energy distribution such as $a, \alpha$, and $\beta$.

Formula (14) enables computation of various relevant observables. Thus, the sputtering yield, $Y\left(\gamma_{0}\right)$, defined as the total number of sputtered atoms per incident ion, is easily related to $v_{O}$ by geometry as $Y\left(\gamma_{0}\right)=$ $n v_{O}\left(\gamma_{0}\right) /\left(\Phi_{0} \cos \gamma_{0}\right)$, where $n$ is the number of atoms per unit volume in the target. Assuming a planar interface, that is, in the $R_{x}, R_{y} \rightarrow \infty$, one is left with

$$
Y\left(\gamma_{0}\right)=\frac{n v_{O}\left(\gamma_{0}, R_{x} \rightarrow \infty, R_{y} \rightarrow \infty\right)}{\Phi_{0} \cos \gamma_{0}} .
$$

Working with Sigmund's distribution, BH found ${ }^{7}$ that $Y\left(\gamma_{0}\right)$ increases monotonously as a function of the indigence angle $\gamma_{0}$, such that the maximum efficiency for erosion is achieved at grazing incidence, contrary to experimental evidence for amorphous, polycrystalline, and crystalline targets ${ }^{31.32 .33}$ This feature of BH's theory originates in a property of Sigmund's distribution (1), whose maximum for deposition, $\mathbf{r}=(0,0,-a)$, is located right at the surface under grazing incidence conditions. However, as is well known, there usually exists a value of $\gamma_{0}<90^{\circ}$ for which the yield is maximum, such that the sputtering efficiency decreases for larger angles of incidence, due to ions being reflected at the surface, an effect which is beyond Sigmund's approximations.

Additional predictions on the morphology of the eroded target can be derived from (14). Thus, $\Gamma_{x}\left(\gamma_{0}\right)$ is negative $e^{\underline{7}}$ for small angles of incidence, which implies that the erosion velocity is larger at troughs $\left(R_{x}<0\right)$ than at peaks $\left(R_{x}>0\right)$, inducing a morphological instability. Additional surface relaxation mechanisms exist, such as surface diffusion, that counteract this instability. Competition between the two opposing phenomena induce the emergence of a typical length scale, associated with the wavelength of the periodic ripple structure appearing (for details, see Sec. VIII). For $\gamma_{0}$ large enough, BH get $\Gamma_{x}>0$, while $\Gamma_{y}$ is always negative. Given that at small angles $\Gamma_{x}<\Gamma_{y}$, one obtains that the ripple crests are oriented perpendicular to the $\hat{\mathbf{x}}^{\prime}$ direction for incidences close to normal, whereas they are oriented perpendicular to the $\hat{\mathbf{y}}^{\prime}$ direction for incidence angles larger than a critical one, $\gamma_{0}>\gamma_{c}$ such that $\Gamma_{y}<\Gamma_{x}$. Many experiments ${ }^{2.34}$ have verified the validity of BH's theory to describe ripple wavelength and orientation. 


\section{MODIFIED ENERGY DISTRIBUTION FUNCTIONS}

The results of computer simulations within the BCA approximation, obtained in the previous sections for $\mathrm{Cu}$ ion bombardment of a $\mathrm{Cu}$ target are described by an energy distribution that differs substantially from that obtained by Sigmund in the case of polycrystalline or amorphous substrates. Using cylindrical coordinates around the ion trajectory, as in previous sections, we have [recall Fig. [5 and Eq. (12) above]

$$
\epsilon_{e}(\boldsymbol{\rho}, z)=N_{e} \epsilon\left(\rho^{2}+c \rho\right) e^{-\frac{\rho}{\sigma_{x y}}} e^{-\frac{(z+a)^{2}}{2 \sigma_{z}^{2}}},
$$

where $N_{e}=\left[(2 \pi)^{3 / 2} \sigma_{z}\left(6 \sigma_{x y}^{4}+2 c \sigma_{x y}^{3}\right)\right]^{-1}$ is a normalization constant. Values for $c$ and $\sigma_{x y}$ that best fit simulation results were $c=-0.392, \sigma_{x y}=0.787$, see Fig. 5. Note two main differences of distribution (16) to Sigmund's distribution (1): decay here is slower [exponential as compared to Gaussian, thus the subscripts in (16)] in the plane perpendicular to the ion trajectory, and energy deposition is null along the ion trajectory itself. On the other hand, distribution (16) is unphysical. since $c<0$ leads to negative probabilities for small $\rho$ values. For this reason, and in order to facilitate analytical results [unavailable for (16) in the physical case of a two dimensional target, see below], we will also consider the following modified Gaussian (thus the subscripts) distribution

$$
\begin{aligned}
\epsilon_{g}(\boldsymbol{\rho}, z) & =N_{g} \epsilon \rho^{2} e^{-\frac{\rho^{2}}{2 \sigma_{x y}^{2}}} e^{-\frac{(z+a)^{2}}{2 \sigma_{z}^{2}}}, \\
N_{g} & =\left[2(2 \pi)^{3 / 2} \sigma_{x y}^{4} \sigma_{z}\right]^{-1},
\end{aligned}
$$

that shares with (16) inducing zero energy deposition along the ion trajectory $\rho=0$, but is otherwise Gaussian in all three directions far enough from the ion path.

\section{A. One-dimensional interfaces}

In order to develop intuition about morphological predictions from (16), (17), that are based on analytical results, we consider first the (non-physical) case of a onedimensional target, whose surface height is described by a single variable function $z^{\prime}\left(x^{\prime}\right)$. These results will be then compared to the analogous ones by Bradley and Harper, which will allow us to assess differences due to the new form of the energy distribution, mostly to the fact that in our case no energy is deposited along ion trajectories.

For a one-dimensional target, distribution (17) reads

$$
\begin{aligned}
\epsilon_{g}^{1 d}(x, z) & =N_{g} \epsilon x^{2} e^{-\frac{x^{2}}{2 \sigma_{x}^{2}}} e^{-\frac{(z+a)^{2}}{2 \sigma_{z}^{2}}}, \\
N_{g}^{1 d} & =\left(2 \pi \sigma_{z} \sigma_{x}^{3}\right)^{-1} .
\end{aligned}
$$

Writing the local velocity of erosion in terms of $\Gamma_{0}^{g, 1 d}$, $\Gamma_{x}^{g, 1 d}$ analogous to Eq. (14), we obtain

$$
v_{O}=N_{g}^{1 d} \Lambda \epsilon \Phi_{0} e^{-\frac{a^{2}}{2 \sigma_{z}^{2}}}\left[\Gamma_{0}^{g, 1 d}+\frac{\Gamma_{x}^{g, 1 d}}{R_{x}}\right],
$$

where the full expressions for $\Gamma_{0}^{g, 1 d}$ and $\Gamma_{x}^{g, 1 d}$ as functions of $\gamma_{0}, a, \sigma_{x}$, and $\sigma_{z}$ can be found in Appendix A1.

On the other hand, distribution (16) reads, for a onedimensional interface,

$$
\begin{aligned}
\epsilon_{e}^{1 d}(x, z) & =N_{e} \epsilon\left(x^{2}+c|x|\right) e^{-\frac{|x|}{\sigma_{x}}} e^{-\frac{(z+a)^{2}}{2 \sigma_{z}^{2}}}, \\
N_{e}^{1 d} & =\left[\sqrt{2 \pi} \sigma_{z}\left(4 \sigma_{x}^{3}+2 c \sigma_{x}^{2}\right)\right]^{-1} .
\end{aligned}
$$

In this case, the prediction for the local velocity of erosion has a shape that is similar to (18), albeit with more complex coefficients, whose detailed analytical expressions are again left to Appendix A2

$$
v_{O}=N_{e}^{1 d} \Lambda \epsilon \Phi_{0} e^{-\frac{a^{2}}{2 \sigma_{z}}}\left[\Gamma_{0}^{e, 1 d}+\frac{\Gamma_{x}^{e, 1 d}}{R_{x}}\right] .
$$

In Fig. 9] we plot the normalized (to the corresponding values for normal incidence) sputtering yields $Y\left(\gamma_{0}\right)$ obtained through (15) for the modified Gaussian (19) and exponential (21) distributions. For the sake of reference, the $\mathrm{BH}$ yield is also shown. We can see that for both modified distributions, (18) and (20), the corresponding yields feature maxima before grazing incidence, as a difference to the $\mathrm{BH}$ curve. This is in agreement with experimental data,, 31.32 .33 and is due to the fact that maxima of energy deposition are not along the ion trajectory for these distributions but, rather, at a certain finite distance from it, what makes grazing incidence not be the most efficient for sputtering. The fact (seen in Fig. 9) that the yield is negative for large incidence angles $\gamma_{0}$, as computed using the exponential distribution, is due to Eq. (20) taking negative values for small distances to the ion path. As will be seen below, this is an artifact of the one-dimensional approximation, as is the fact that the yield computed from the modified Gaussian distribution (18) vanishes for $\gamma_{0}=90^{\circ}$. From the figure we can also conclude that qualitative behaviors of distributions (18) and (20) are similar, the advantage of the first one being its greater analytical simplicity. Parameters employed in Fig. 9 are typical for $\mathrm{Cu}$ ion bombardment of $\mathrm{Cu}$ for energies in the range of a few $\mathrm{keV}$, as confirmed by TRIM/SRIM simulations ${ }^{20}$

\section{B. Two-dimensional interfaces}

Naturally, the physically relevant case is bombardment of two-dimensional targets. In this case, the analysis is more complex, to the extreme that no closed expressions analogous of those found previously for the exponential distribution (16) that best fits our BCA simulation data. Results for this distribution will be provided from numerical solutions of (13) using (16). On the other hand, we have seen in the $1 \mathrm{~d}$ case that distributions (18) and (20) lead to similar qualitative results. For the $2 \mathrm{~d}$ case, expression (13) using (17) leads to closed analytical expressions for the coefficients in

$$
v_{O}=N_{g} \Lambda \epsilon \Phi_{0} e^{-\frac{a^{2}}{2 \sigma_{z}^{2}}}\left[\Gamma_{0}^{g}+\frac{\Gamma_{x}^{g}}{R_{x}}+\frac{\Gamma_{y}^{g}}{R_{y}}\right],
$$




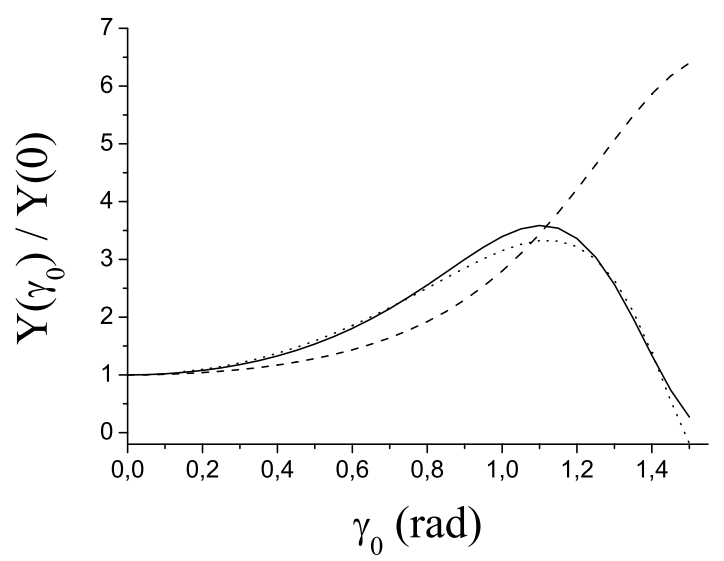

FIG. 9: Normalized sputtering yield $Y\left(\gamma_{0}\right) / Y(0)$ as a function of incidence angle $\gamma_{0}$, for the various one-dimensional energy distributions. Dashed line: Bradley-Harper for $a=3.8$ $\mathrm{nm}, \alpha=2.2 \mathrm{~nm}, \beta=1.5 \mathrm{~nm}$. Solid line: modified Gaussian, Eq. (18), for $a=3.8 \mathrm{~nm}, \sigma_{z}=2.2 \mathrm{~nm}, \sigma_{x}=1.5 \mathrm{~nm}$. Dotted line: exponential, Eq. (20), for $a=3.8 \mathrm{~nm}, \sigma_{z}=2.2 \mathrm{~nm}$, $\sigma_{x}=0.787 \mathrm{~nm}, c=-0.392 \mathrm{~nm}$.

that can be found in Appendix A3 In Fig. 10 we see again that the sputtering yield for both modified distributions (16) and (17) have maxima for incidence angles clearly smaller than grazing. Moreover, the yields are positive and non-zero for all values of $\gamma_{0}$, and amount to large sputtering rates, as found in experiments ${ }^{31.32 .33}$. In the present two-dimensional case, for grazing incidence the radial component of the energy distribution vanishes at the point of impact with the surface, but not at finite distances from it, which implies that after surface integration the total deposited energy is non-zero and the yield is positive. Again, Fig. 10] shows similar qualitative behaviors for both modified distributions, the modified Gaussian having the advantage of leading to closed analytical results. For normal incidence, the $x \leftrightarrow y$ symmetry is restored, and thus $\Gamma_{x}\left(\gamma_{0}\right)$ and $\Gamma_{y}\left(\gamma_{0}\right)$ must coincide. From Eq. (22), we obtain $\Gamma_{x}^{g}(0)=\Gamma_{y}^{g}(0)=-4 \pi a \sigma_{x y}^{6} / \sigma_{z}^{2}$, whose coincidence with the numerical results for distribution (16) has been confirmed. In Figs. 11] 12 and 13 we present results for the "surface tension" coefficients $\Gamma_{x}$ and $\Gamma_{y}$ for the various two-dimensional distributions, that are normalized by their corresponding absolute values for normal incidence $\gamma_{0}=0$. We see in Figs. 12, 13 that $\Gamma_{x}$ is smaller than $\Gamma_{y}$ for incidence angles $\gamma_{0}<\gamma_{c}$ and that $\Gamma_{y}$ is always negative, similarly to the $\mathrm{BH}$ case (Fig. 11). One of the successes of BH's theory lies in its description of the orientation of the ripple structure for different ion incidence angles $\gamma_{0}$. Here we see that, although distributions (16) and (17), lead to quite different sputtering yields as compared to Sigmund's distribution, the qualitative behavior of coefficients $\Gamma_{x}$ and $\Gamma_{y}$ is quite similar to that found by BH. Since experimental results are in good agreement with BH for metals, the modified Gaussian distribution (17) seems a good choice for the

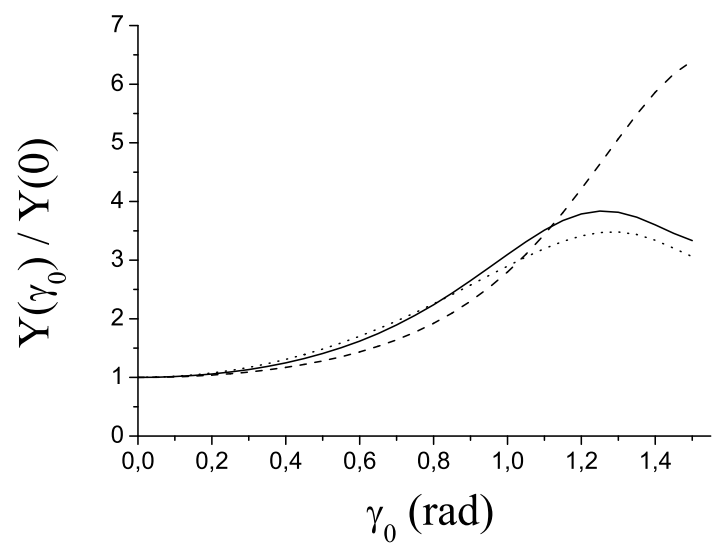

FIG. 10: Normalized sputtering yield $Y\left(\gamma_{0}\right) / Y(0)$ as a function of incidence angle $\gamma_{0}$, for the various two-dimensional energy distributions. Dashed line: Bradley-Harper, Eq. (1), for $a=3.8 \mathrm{~nm}, \alpha=2.2 \mathrm{~nm}, \beta=1.5 \mathrm{~nm}$. Solid line: modified Gaussian, Eq. (17), for $a=3.8 \mathrm{~nm}, \sigma_{z}=2.2 \mathrm{~nm}, \sigma_{x y}=1.5$ $\mathrm{nm}$. Dotted line: exponential, Eq. (16), for $a=3.8 \mathrm{~nm}$, $\sigma_{z}=2.2 \mathrm{~nm}, \sigma_{x}=0.787 \mathrm{~nm}, c=-0.392 \mathrm{~nm}$.

corresponding analytical description, with the advantage over the exponential distribution (16) of being physically sound for small distances to the point of penetration 3.4

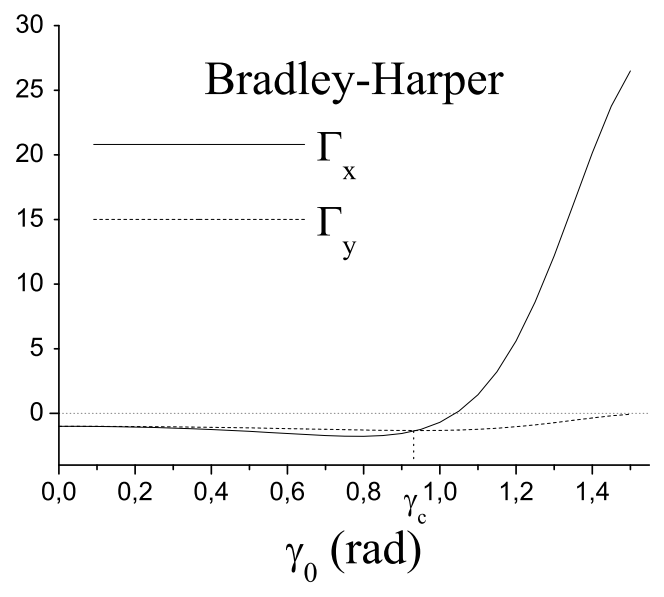

FIG. 11: Normalized values of $\Gamma_{x}$ and $\Gamma_{y}$ for the distribution (1) using the same parameter values as in Fig. 10]

\section{CONTINUUM EQUATION FOR THE SURFACE HEIGHT}

Following the pioneering approach by Bradley and Harper, we can derive an evolution (differential) equation for the surface height, starting from the equation for the erosion velocity. We consider a laboratory frame of reference $(\hat{\mathbf{X}}, \hat{\mathbf{Y}}, \hat{\mathbf{Z}})$, defined as follows: the $\hat{\mathbf{Z}}$ axis is chosen 


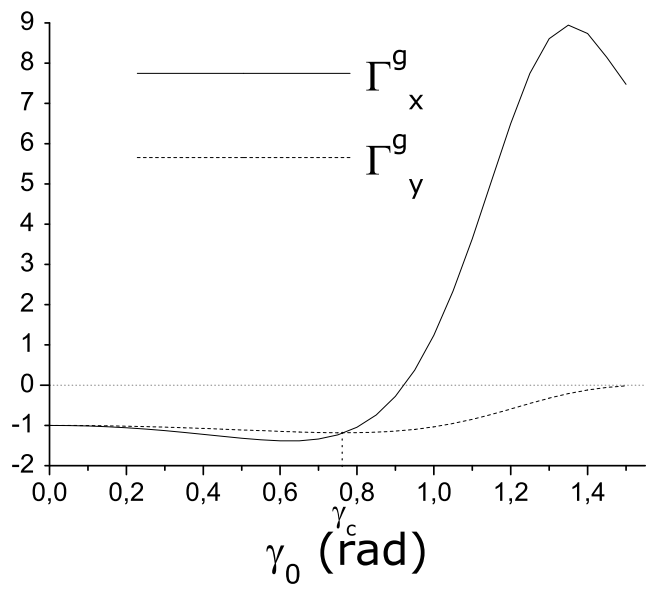

FIG. 12: Normalized values of $\Gamma_{x}^{g}$ and $\Gamma_{y}^{g}$ for the distribution (17) using the same parameter values as in Fig. 10]

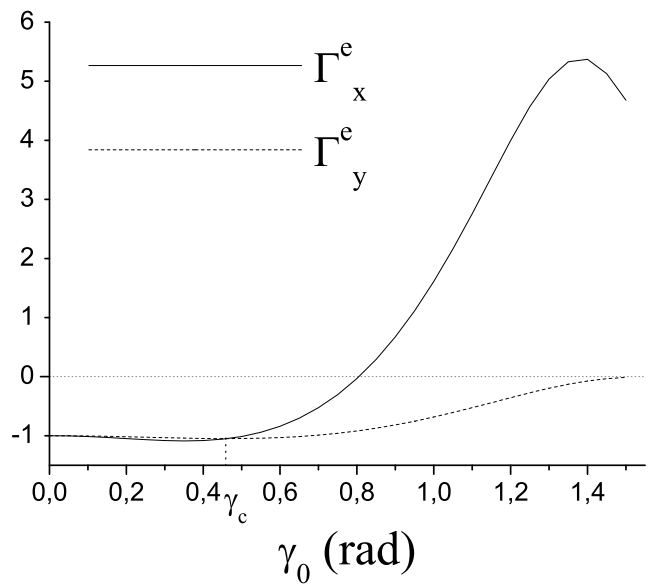

FIG. 13: Normalized values of $\Gamma_{x}^{e}$ and $\Gamma_{y}^{e}$ for the distribution (16) using the same parameter values as in Fig. 10]

to be normal to the initial planar surface. The incoming beam direction forms an angle $\theta$ with $\hat{\mathbf{Z}}$, and both direction define a plane where the $\hat{\mathbf{X}}$ axis lies. Finally, the $\hat{\mathbf{Y}}$ axis is perpendicular to the $\hat{\mathbf{X}}$ and $\hat{\mathbf{Z}}$ directions. We describe by $h(X, Y, t)$ the surface height at time $t$ above point $(X, Y)$ on reference plane of the unbombarded substrate, and assume that it varies slowly enough so we can work to first order in the derivates. In this way we may approximate: ${ }^{7.8 .9} \gamma_{0}=\theta-\frac{\partial h}{\partial X}, \frac{1}{R_{x}}=-\frac{\partial^{2} h}{\partial X^{2}}, \frac{1}{R_{y}}=-\frac{\partial^{2} h}{\partial Y^{2}}$. The velocity of erosion of the surface height $h$ is provided by the erosion rate $v_{O}$, and we thus get:

$$
\frac{1}{F} \frac{\partial h}{\partial t} \cong-\Gamma_{0}(\theta)+\frac{\partial \Gamma_{0}(\theta)}{\partial \theta} \frac{\partial h}{\partial X}+\Gamma_{x} \frac{\partial^{2} h}{\partial X^{2}}+\Gamma_{y} \frac{\partial^{2} h}{\partial Y^{2}},
$$

where in our normalization $F$ is a proportionality constant between $v_{O}$ and $\Gamma_{0}, \Gamma_{x}, \Gamma_{y}$, that can be found in the Appendix. Considering a periodic perturbation to the planar surface $h(X, Y, t=0)=A e^{i\left(k_{1} X+k_{2} Y\right)}$, and substi- tuting this expression into Eq. (23), the surface profile evolves as

$$
\begin{aligned}
& h(X, Y, t)=-\Gamma_{0} t+A e^{r t} e^{i\left(k_{1} X+k_{2} Y-\omega t\right)}, \\
& r=-\Gamma_{x} k_{1}^{2}-\Gamma_{y} k_{2}^{2}, \\
& \omega=-\Gamma_{0}^{\prime} k_{1} .
\end{aligned}
$$

If $\Gamma_{x}$ and/or $\Gamma_{y}$ are negative, there will be values for the wave-vector $\left(k_{1}, k_{2}\right)$ of the perturbation that make it grow exponentially. This behavior is a reflection of the well-known physical instability leading to ripple formation,, .30 due to the curvature dependence of the erosion velocity, that is larger in surface troughs than in surface protrusions. The observed ripple wavelength arises when additional smoothing mechanims such as surface diffusion exist that compete with the sputter instability, leading to selection of a specific length-scale. Taking these mechanisms into account,, .9 Eq. (23) reads

$$
\begin{aligned}
\frac{\partial h}{\partial t} \cong & F\left\{-\Gamma_{0}(\theta)+\frac{\partial \Gamma_{0}(\theta)}{\partial \theta} \frac{\partial h}{\partial X}+\Gamma_{x} \frac{\partial^{2} h}{\partial X^{2}}+\Gamma_{y} \frac{\partial^{2} h}{\partial Y^{2}}\right\} \\
& -B \nabla^{4} h,
\end{aligned}
$$

where, in principle, $B$ is a thermally activated coefficient which depends on the surface self-diffusivity $D_{s}$, the free energy per unit area $\gamma$ and the number of atoms per unit area moving across the surface $\sigma$ as $B=2 D_{s} \gamma \sigma /\left(n^{2} k_{B} T\right)$. In this case, $r=-\Gamma_{x} k_{1}^{2}-\Gamma_{y} k_{2}^{2}-$ $B\left(k_{1}^{2}+k_{2}^{2}\right)^{2}$, and there is only a band of unstable perturbations. The observed ripple wavelength $\ell$ is provided by the wave-vector which has the largest positive value of $r$, and is proportional to $\sqrt{\frac{B}{F\left|\Gamma_{x}\right|}}$ or $\sqrt{\frac{B}{F\left|\Gamma_{y}\right|}}$ when $\Gamma_{x}<\Gamma_{y}<0\left(\theta<\theta_{c}\right)$ or $\Gamma_{y}<\Gamma_{x}\left(\theta>\theta_{c}\right)$, respectively.

As we have seen in the previous section, the behavior of $\Gamma_{x}$ and $\Gamma_{y}$ is similar for all the cases considered, while the qualitative behavior of the yield is quite different to that found by Bradley and Harper for amorphous or policrystalline substrates. Moreover, since dependences of the ripple wavelength $\ell \propto \sqrt{\frac{B}{F|\Gamma|}}$ on parameters such as ion flux, $\Phi_{0}$, temperature, or average ion energy, $\epsilon$, are due to those in the constants $F$ and $B$, and these are the same as those in BH (see Appendix), Eq. (25) predicts these for $\mathrm{Cu}$ to be (qualitatively) the same as obtained from $\mathrm{BH}$ theory $\frac{9}{}$ Note this is also the case in the presence of non-thermal surface diffusion, in which, similarly to $\mathrm{BH}, \frac{9}{9}$ the constant $B$ has no dependence on temperature and is, rather, proportional to $F$.

\section{SUMMARY AND OUTLOOK}

We have studied numerically the sputtering process of $\mathrm{Cu}$ ions on $\mathrm{Cu}$ fcc crystals by means of the binary collision approximation. We have analyzed the distribution of sputtered particles and their energies, and found significant deviations from Sigmund's formula, which is traditionally employed to study the sputtering process 
in the framework of continuum theories, as applied to amorphous and policrystalline substrates. In particular, we find that near the point where the ion penetrates the target, the sputter probability goes to zero, while the Bradley-Harper/Sigmund theory predicts maximum sputtering at that point.

We have fitted heuristic functions to our data. We find that an exponential (rather than Gaussian as in Sigmund's theory) decay with a combination of a quadratic and a linear prefactor fits the data well. The main physical effect, namely, the "hole" near the point of penetration, can be reproduced also qualitatively using a Gaussia distribution with with a quadratic prefactor, that is physically better defined than the heuristic fitting distribution, and lends itself to exact results. We have performed analytical calculations of the local erosion velocity following the Bradley-Harper approach for one- and two-dimensional surfaces, for both types of modified distributions (for the two-dimensional exponential distribution, the equation could be solved only numerically). We find that the sputter yield is qualitatively different as compared the the $\mathrm{BH}$ approach. As a function of the angle of incidence, the yield exhibits a maximum at an intermediate angle, and then decreases when approaching grazing incidence. This is in good agreement with experimental findings, in marked contrast with the analogous BH result using Sigmund's distribution, even without implementing explicitly reflection of the ions for grazing incidence, which is usually regarded as the main cause for the decay of the yield at grazing incidence. Finally, we have computed also the ripple orientation-determining parameters $\Gamma_{x}, \Gamma_{y}$, usually referred to in this context as effective surface tension parameters. These turn out to be only slightly modified with respect to the $\mathrm{BH}$ theory, and lead to a qualitatively similar pattern formation process. Dependencies of the ripple wavelength on phenomenological parameters, such as ion flux, ion average energy, and temperature are as in $\mathrm{BH}$ theory ${ }^{9}$ Since the influence of non-linearities on ripple characteristics is still under debate even within Sigmund's theory proper, we have not considered this type of effects here. At any rate, the same type of non-linear terms would appear in the interface Eq. (25) as compared to the corresponding equation for amorphous or policrystalline substrates $\frac{8.9}{.9}$

Thus, as a general conclusion on pattern formation by ion-beam sputtering, our results justify the similarities found in experiments on metals, to the analogous processes in amorphous or amorphizable materials, and point to potential quantitative differences that would possibly merit further studies. Additional features of ripple formation in metals, such as their existence for normal incidence or change of orientation with temperature ${ }^{3,15,16}$ are not explained by the special properties of the collision cascades in these systems that we have studied here but, rather, by the special properties of surface diffusion in such anisotropic substrates.

Regarding future work, it would be also interesting to see whether the hole near the point of penetration can be found in experiments, and/or in more detailed simulations (such as e.g. by Molecular Dynamics). To our knowledge, no analysis of single-ion impacts on metals exist so far. Furthermore, it would be worth incorporating the modified energy distribution into existing simple Monte Carlo models of surface sputtering, such as those in Refs. 17, 35, in order to improve their description of erosion processes in metallic substrates, specially at the large distance and long time regime for which this type of models is particularly suited.

\section{Acknowledgments}

This work has been supported by the Sonderforschungsbeeich 602 of the Deutsche Forschungsgemeinschaft, and by MECD (Spain) grant No. BFM200307749-C05-01. A. K. H. obtained financial support from the VolkswagenStiftung (Germany) within the program "Nachwuchsgruppen an Universitäten". A. K. H. thanks K.P. Lieb for helpful suggestions. J. M.-G. acknowledges support from MECD (Spain) through an FPU fellowship.

\section{APPENDIX A: ANALYTIC EXPRESSIONS FOR COEFFICIENTS IN THE EROSION VELOCITY}

In this appendix, we provide the full expressions for the coefficients appearing in various expressions for the surface erosion velocity, Eqs. (21), (19), and (22), that have been computed analytically for those energy distributions for which such type of results are achievable.

\section{One-dimensional modified Gaussian distribution}

$$
\begin{gathered}
v_{O}=N_{g}^{1 d} \Lambda \epsilon \Phi_{0} e^{-\frac{a^{2}}{2 \sigma_{z}^{2}}}\left[\Gamma_{0}^{g, 1 d}+\frac{\Gamma_{x}^{g, 1 d}}{R_{x}}\right] \\
\Gamma_{0}^{g, 1 d}=\frac{\sqrt{\pi} e^{\frac{A_{g}^{2}}{4 B_{g}}}\left(A_{g}^{2}+2 B_{g}\right)}{4 B_{g}^{5 / 2}} \cos ^{3} \gamma_{0} \\
\Gamma_{x}^{g, 1 d}=\frac{\sqrt{\pi} e^{\frac{A_{g}^{2}}{4 B_{g}}}}{32 B_{g}^{11 / 2}}\left[2 A_{g}^{3} B_{g}\left(A_{g} b_{g}-10 c_{g}\right)-A_{g}^{5} c_{g}-\right. \\
\left.4 A_{g} B_{g}^{2}\left(A^{2} a_{g}-6 A_{g} b_{g}+15 c_{g}\right)-24 B_{g}^{3}\left(A_{g} a_{g}-b_{g}\right)\right] \\
A_{g}=\frac{a}{\sigma_{z}^{2}} \sin \gamma_{0}, B_{g}=\frac{1}{2 \sigma_{z}^{2}} \sin ^{2} \gamma_{0}+\frac{1}{2 \sigma_{x}^{2}} \cos ^{2} \gamma_{0} \\
a_{g}=-2 \sin \gamma_{0} \cos ^{2} \gamma_{0}, b_{g}=-\frac{a}{2 \sigma_{z}^{2}} \cos ^{4} \gamma_{0} \\
c_{g}=\left(\frac{1}{2 \sigma_{x}^{2}}-\frac{1}{2 \sigma_{z}^{2}}\right) \cos ^{4} \gamma_{0} \sin _{0} .
\end{gathered}
$$




\section{One-dimensional exponential distribution}

$$
\begin{aligned}
& v_{O}=N_{e}^{1 d} \Lambda \epsilon \Phi_{0} e^{-\frac{a^{2}}{2 \sigma_{z}^{2}}}\left[\Gamma_{0}^{e, 1 d}+\frac{\Gamma_{x}^{e, 1 d}}{R_{x}}\right] \\
& \Gamma_{0}^{e, 1 d}=\frac{a_{e}}{B_{e}}+\frac{1}{8 B_{e}^{5 / 2}} \sum_{i=1,2}\left\{-2 \sqrt{B_{e}} A_{e, i} b_{e}+\right. \\
& \left.\left[A_{e, i}^{2} b_{e}-2 a_{e} A_{e, i} B_{e}+2 b_{e} B_{e}\right] \sqrt{\pi} e^{\frac{A_{e, i}^{2}}{4 B_{e}}} \operatorname{erfc}\left(\frac{A_{e, i}}{2 \sqrt{B_{e}}}\right)\right\} \\
& \Gamma_{x}^{e, 1 d}=\frac{1}{64 B_{e}^{11 / 2}} \sum_{i=1,2}\left\{2 \sqrt { B _ { e } } \left[(-1)^{i} A_{e, i}^{4} f_{e}-2 A_{e, i}^{3} B_{e} e_{e, i}+\right.\right. \\
& +(-1)^{i} 16 B_{e}^{3} d_{e, i}-4 B_{e}^{2}\left(A_{e, i}\left(5 e_{e, i}+(-1)^{i} 2 B_{e} c_{e}\right)\right)+ \\
& \left.(-1)^{i} 18 A_{e, i}^{2} B_{e} f_{e}+(-1)^{i} 4 A_{e, i} B_{e}^{2} d_{e, i}\right]+ \\
& {\left[-(-1)^{i} A_{e, i}^{5} f_{e}+2 A_{e, i}^{3} B_{e}\left(A_{e, i} e_{e, i}-(-1)^{i} 10 f_{e}\right)+\right.} \\
& -4 A_{e, i} B_{e}^{2}\left((-i)^{i} A_{e, i}^{2} d_{e, i}-6 A_{e, i} e_{e, i}+(-1)^{i} 15 f_{e}\right)+ \\
& \left.8 B_{e}^{3}\left((-1)^{i} A_{e, i}^{2} c_{e}-(-1)^{i} 3 A_{e, i} d_{e, i}+3 e_{e, i}\right)\right] \times \\
& \left.\sqrt{\pi} e^{\frac{A_{e, i}^{2}}{4 B_{e}}} \operatorname{erfc}\left(\frac{A_{e, i}}{2 \sqrt{B_{e}}}\right)\right\} \\
& A_{e, 1}=\frac{\cos \gamma_{0}}{\sigma_{x}}-\frac{a \sin \gamma_{0}}{\sigma_{z}^{2}}, A_{e, 2}=\frac{\cos \gamma_{0}}{\sigma_{x}}+\frac{a \sin \gamma_{0}}{\sigma_{z}^{2}} \\
& B_{e}=\frac{\sin ^{2} \gamma_{0}}{2 \sigma_{s}^{2}}, a_{e}=c \cos ^{2} \gamma_{0}, b_{e}=\cos ^{3} \gamma_{0} \\
& c_{e}=-\frac{3}{2} c \cos \left(\gamma_{0}\right) \sin \left(\gamma_{0}\right) \\
& d_{e, 1}=\left(\frac{c}{2 \sigma_{x}}-2\right) \cos ^{2}\left(\gamma_{0}\right) \sin \left(\gamma_{0}\right)+\frac{c a}{2 \sigma_{z}^{2}} \cos ^{3}\left(\gamma_{0}\right), \\
& d_{e, 2}=\left(\frac{c}{2 \sigma_{x}}-2\right) \cos ^{2}\left(\gamma_{0}\right) \sin \left(\gamma_{0}\right)-\frac{c a}{2 \sigma_{z}^{2}} \cos ^{3}\left(\gamma_{0}\right), \\
& e_{e, 1}=\left(\frac{c}{2 \sigma_{z}^{2}}-\frac{1}{2 \sigma_{x}}\right) \cos ^{3}\left(\gamma_{0}\right) \sin \left(\gamma_{0}\right)-\frac{a}{2 \sigma_{z}^{2}} \cos ^{4}\left(\gamma_{0}\right) \text {, } \\
& e_{e, 2}=-\left(\frac{c}{2 \sigma_{z}^{2}}+\frac{1}{2 \sigma_{x}}\right) \cos ^{3}\left(\gamma_{0}\right) \sin \left(\gamma_{0}\right)-\frac{a}{2 \sigma_{z}^{2}} \cos ^{4}\left(\gamma_{0}\right), \\
& f_{e}=2 \cos ^{5}\left(\gamma_{0}\right) \sin \left(\gamma_{0}\right)
\end{aligned}
$$

\section{Two-dimensional modified Gaussian distribution}

$$
\Gamma_{0}^{g}=\frac{\pi e^{\frac{A_{g}^{2}}{4 B_{g}}}\left(b_{0} A_{g}^{2}+4 a_{0} B_{g}^{2}+2 b_{0} B_{g}\right)}{2 \sqrt{2} B_{g}^{5 / 2}}
$$

$$
\begin{aligned}
\Gamma_{x}^{g} & =\frac{\pi e^{\frac{A_{g}^{2}}{4 B_{g}}}}{16 \sqrt{2} B_{g}^{11 / 2}}\left\{2 B _ { g } \left[4 B_{g}^{2} b_{x}\left(A_{g}^{2}+2 B_{g}\right)-8 A_{g} B_{g}^{3} a_{x}+\right.\right. \\
& \left.-2 A_{g} B_{g} c_{x}\left(A_{g}^{2}+6 B_{g}\right)+d_{x}\left(A_{g}^{4}+12 A_{g}^{2} B_{g}+12 B_{g}^{2}\right)\right]+ \\
& \left.-A_{g} e_{x}\left(A_{g}^{4}+20 A_{g}^{2} B_{g}+60 B_{g}^{2}\right)\right\}
\end{aligned}
$$

$$
\begin{gathered}
\Gamma_{y}^{g}=\frac{\pi e^{\frac{A_{g}^{2}}{4 B_{g}}}}{4 \sqrt{2} B_{g}^{7 / 2}}\left[2 B_{g} c_{y}\left(A_{g}^{2}+2 B_{g}\right)-4 B_{g}^{2} a_{y}\left(b_{y}-2 B_{g}\right)+\right. \\
\left.-A_{g} d_{y}\left(A_{g}^{2}+6 B_{g}\right)\right] \\
A_{g}=\frac{a}{\sigma_{z}^{2}} \sin \gamma_{0}, B_{g}=\frac{1}{2 \sigma_{z}^{2}} \sin ^{2} \gamma_{0}+\frac{1}{2 \sigma_{x y}^{2}} \cos ^{2} \gamma_{0} \\
a_{0}=\sigma_{x y}^{3} \cos \gamma_{0}, b_{0}=\sigma_{x y} \cos ^{3} \gamma_{0}
\end{gathered}
$$

$$
a_{x}=-\sigma_{x y}^{3} \sin \gamma_{0}, b_{x}=-\frac{\sigma_{x y}^{3}}{2 \sigma_{z}^{2}} a \cos ^{2} \gamma_{0}
$$$$
c_{x}=-\left(\frac{3}{2} \sigma_{x y}+\frac{\sigma_{x y}^{3}}{2 \sigma_{z}^{2}}\right) \cos ^{2} \gamma_{0} \sin \gamma_{0}
$$

$$
d_{x}=-\frac{\sigma_{x y}}{2 \sigma_{z}^{2}} a \cos ^{4} \gamma_{0}, e_{x}=\left(\frac{1}{2 \sigma_{x y}}-\frac{\sigma_{x y}}{2 \sigma_{z}^{2}}\right) \sin \gamma_{0} \cos ^{4} \gamma_{0}
$$$$
a_{y}=-\frac{3 \sigma_{x y}^{5}}{2 \sigma_{z}^{2}} a \cos ^{2} \gamma_{0}
$$

$$
b_{y}=-\frac{3 \sigma_{x y}^{5}}{2 \sigma_{z}^{2}} \cos ^{2} \gamma_{0} \sin \gamma_{0}+\frac{\sigma_{x y}^{3}}{2} \cos ^{2} \gamma_{0} \sin \gamma_{0}
$$

$$
c_{y}=-\frac{\sigma_{x y}^{3}}{2 \sigma_{z}^{2}} a \cos ^{4} \gamma_{0}
$$

$$
d_{y}=\left(\frac{\sigma_{x y}}{2}-\frac{\sigma_{x y}^{3}}{2 \sigma_{z}^{2}}\right) \cos ^{4} \gamma_{0} \sin \gamma_{0}
$$

$$
v_{O}=N_{g} \Lambda \epsilon \Phi_{0} e^{-\frac{a^{2}}{2 \sigma_{z}^{2}}}\left[\Gamma_{0}^{g}+\frac{\Gamma_{x}^{g}}{R_{x}}+\frac{\Gamma_{y}^{g}}{R_{y}}\right]
$$


* hartmann@theorie.physik.uni-goettingen.de

† reiner.kree@physik.uni-goettingen.de

¥ jamunoz@math.uc3m.es

$\S$ cuerno@math.uc3m.es

1 G. W. Lewis, M. J. Nobes, G. Carter, and J. L. Whitton, Nucl. Instrum. Methods 179, 363 (1980).

2 E. Chason, T. M. Mayer, B. K. Kellermann, D. E. McIlroy, and A. J. Howard, Phys. Rev. Lett. 72, 3040 (1994).

3 S. Rusponi, G. Constantini, C. Boragno, and U. Valbusa, Phys. Rev. Lett. 81, 4184 (1998).

4 S. Habenicht, W. Bolse, K. P. Lieb, K. Reimann, and U. Geyer, Phys. Rev. B 60, R2200 (1999).

5 T. M. Mayer, E. Chason, and A. J. Howard, J. Appl. Phys. 76, 1633 (1994).

6 U. Valbusa, C. Boragno, and F. B. de Mongeot, J. Phys.: Condens. Matter 14, 8153 (2002).

7 R. M. Bradley and J. M. E. Harper, J. Vac. Sci. Technol A 6, 2390 (1988).

8 R. Cuerno and A. L. Barabási, Phys. Rev. Lett. 74, 4746 (1995).

9 M. Makeev, R. Cuerno, and A. L. Barabási, Nucl. Inst. and Meth. Phys. Res. B 197, 185 (2002).

10 C. M. Demanet, J. B. Malherbe, N. G. Vanderberg, and V. Sankar, Surf. Interface Anal. 23, 433 (1995).

11 S. W. MacLaren, J. E. Baker, N. L. Finnegan, and C. M. Loxton, J. Vac. Sci. Technol. A 10, 468 (1992).

12 G. Carter and V. Vishnyakov, Phys. Rev. B 54, 17647 (1996).

13 J. Malherbe, CRC Crit. Rev. Solid State Mater. Sci. 19, 55 (1994), and references therein.

14 J. Erlebacher, A. J. Aziz, E. Chason, M. B. Sinclair, and J. A. Floro, Phys. Rev. Lett. 82, 2330 (1999).

15 S. Rusponi, C. Boragno, and U. Valbusa, Phys. Rev. Lett. 78, 2795 (1997).

16 S. Rusponi, G. Constantini, C. Boragno, and U. Valbusa,
Phys. Rev. Lett. 81, 2735 (1998).

17 A. K. Hartmann, R. Kree, U. Geyer, and M. Kölbel, Phys. Rev. B 65, 193403 (2002).

18 E. O. Yewande, A. K. Hartmann, and R. Kree (2004), submitted to Phys. Rev. B, preprint cond-mat/0405363.

19 P. Sigmund, Phys. Rev. 184, 383 (1969).

20 J. F. Ziegler, J. P. Biersack, and U. Littmark, The Stopping and Range of Ions in Matter (Pergamon, New York, 1985).

21 T. A. Tombrello, Nucl. Instr. and Meth. Phys. Res. B 83, 508 (1993).

22 M. T. Robinson and I. M. Torrens, Phys. Rev. B 9, 5008 (1974).

23 I. Koponen, M. Hautala, and O. P. Sievänen, Phys. Rev. Lett. 78, 2612 (1997).

24 M. T. Robinson, Rad. Effects and Defects in Solids 130, 3 (1994).

25 I. Koponen, M. Hautala, and O. P. Sievänen, Phys. Rev. B 54, 13502 (1996).

26 G. Moliere, Z. Naturforschung A 2, 133 (1947).

27 O. B. Firsov, Sov. Phys. JETP 36, 1076 (1959).

28 We performed also some test simulations for Ar ions and obtained qualitatively the same results. Hence, we show only the results for $\mathrm{Cu}$ ions here.

${ }^{29}$ H. C. Hofsaess (2004), private communication.

30 P. Sigmund, J. Mat. Sci. 8, 1545 (1973).

31 H. Oechsner, Appl. Phys. 8, 185 (1975).

${ }^{32}$ K. B. Cheney and E. T. Pitkin, 36, 3542 (1965).

33 A. Olivia-Florio, R. A. Baragiola, M. M. Jakas, E. V. Alonso, and J. Ferrón, Phys. Rev. B 35, 2198 (1987).

34 J. J. Vajo, R. E. Doty, and E. H. Cirlin, J. Vac. Sci. Technol. A 14, 2709 (1996).

35 R. Cuerno, H. A. Makse, S. Tomassone, S. T. Harrington, and H. E. Stanley, Phys. Rev. Lett. 75, 4464 (1995). 